\title{
Análisis biomecánico en la zona para-radicular de primer molar con y sin la aplicación de micro-osteoperforaciones en mandíbula. Estudio en Elementos Finitos.
}

\author{
Para-root zone biomechanical analysis with and without the \\ application of micro-osteoperforations in the jaw: Finite Elements \\ Study.
}

\author{
Carolina Minte $^{1}$; Eduardo Borie ${ }^{2}$; Eduardo Leal ${ }^{3}$; Paulo Sandoval ${ }^{4 *}$
}

\begin{abstract}
1. Alumna Programa Magister en Odontología, Universidad de La Frontera, Temuco, Chile.

2. Centro Investigación CICO, Universidad de La Frontera, Temuco, Chile.

3. Departamento Ingeniería Mecánica, Universidad de La Frontera, Temuco, Chile.

4. Departamento de Odontopediatría y Ortodoncia, Universidad de La Frontera, Temuco, Chile.
\end{abstract}

* Correspondencia Autor: Paulo Sandoval | Direccion: Avda Francisco Salazar 01145, Temuco, Chile. | Teléfono: +45 2955977 | Teléfono: paulo. sandoval@ufrontera.cl.

Trabajo recibido el 26/10/2020

Trabajo revisado 21/01/2021

Aprobado para su publicación el 11/02/2021

\begin{abstract}
RESUMEN
Objetivo: El propósito del presente estudio fue investigar biomecánicamente si el tratamiento con micro-osteoperforaciones (Mops) genera diferencias de desplazamiento y de tensiones a nivel óseo cuando se aplica una carga ortodóncica, mediante el uso de análisis de elementos finitos. Material y Método: Un modelo de mandíbula dentada donde se eliminó el segundo premolar fue utilizado para el análisis. Posteriormente, se dividieron en 3 muestras dependiendo de la posición de las Mops: 1) Sin Mops (control); 2) Mops $1 \mathrm{~mm}$ adyacentes al primer molar; 3) Mops a $4 \mathrm{~mm}$ del molar. Para la simulación, se aplicó una carga estática horizontal de $150 \mathrm{gr}(1,5 \mathrm{~N})$, simulando un resorte cerrado de Nitinol, tanto a nivel molar en dirección mesial como a nivel interproximal entre canino e incisivo lateral en dirección distal. Resultados: A pesar que se observó una ligera tendencia a aumentar el desplazamiento del molar con la presencia de Mops, no existieron mayores variaciones en relación a las magnitudes de desplazamiento ni tensiones entre los diferentes modelos. Conclusiones: Desde el punto de vista biomecánico no existen diferencias evidentes en los valores de tensiones ni de desplazamiento entre los modelos analizados.
\end{abstract}

PALABRAS CLAVE

Micro-osteoperforaciones; Análisis elementos finitos; Carga ortodóncica.

Int. J. Inter. Dent Vol. 14(2); 162-164, 2021.

\begin{abstract}
Objective: The purpose of the present study was to biomechanically investigate if the treatment with micro-osteoperforations (Mops) generates displacement and tensions differences at bone level when an orthodontic load is applied, through the use of finite element analysis. Material and Method: A toothed jaw model where the second premolar was removed was used for the analysis. Subsequently, they were divided into 3 samples depending on the position of the Mops: 1) Without Mops (control); 2) Mops adjacent $1 \mathrm{~mm}$ to the first molar; 3 ) Mops $4 \mathrm{~mm}$ to molar. To simulate a closed Nitinol spring, an horizontal static load of $150 \mathrm{gr}(1.5 \mathrm{~N})$ was applied, both at molar level in the mesial direction and at interproximal level between the canine and the lateral incisor in the distal direction. Results: Although a slight tendency to increase the displacement of the molar with the presence of Mops was observed, there were no major variations in relation to the magnitudes of displacement or tensions between the different models. Conclusions: From the biomechanical point of view, there are no obvious differences in the values of stresses or displacement between the models analyzed.

KEY WORDS
\end{abstract}

Micro-osteoperforations; Finite elements analysis; Orthodontic load.

Int. J. Inter. Dent Vol. 14(2); 162-164, 2021.

\section{INTRODUCCIÓN}

El movimiento dental en ortodoncia, producido por la aplicación de fuerzas mecánicas, es el resultado de una actividad dinámica de aposición y reabsorción ósea ${ }^{(1)}$, siendo la modelación ósea y su remodelación determinantes para el movimiento dental. Tres tipos de células óseas juegan un rol significativo en la biología del movimiento dentario: osteoblastos, osteocitos y osteoclastos ${ }^{(2)}$.

Las fuerzas ortodóncicas producen diferentes tipos de movimiento dependiendo de la magnitud y dirección de la fuerza aplicada a los dientes. Cada tipo de movimiento dentario es causado por un estrés específico distribuido alrededor del ligamento periodontal y hueso alveolar. Es aceptado que las áreas que experimentan el mayor estrés compresivo presentan los mayores niveles de reabsorción ósea 
osteoclástica(3). Las fuerzas ortodóncicas pueden tener diferentes orígenes, pero inicialmente todas producen una respuesta inflamatoria aséptica aguda, con una temprana liberación de quimiocinas por parte de las células locales. Estas son pequeñas proteínas capaces de atraer células distantes a la zona, facilitando la expresión de moléculas de adhesión en los vasos sanguíneos y estimulando el reclutamiento de células inflamatorias y precursoras desde la microvascularización hacia el espacio extravascular ${ }^{(4)}$

Para satisfacer la creciente demanda de tratamientos de ortodoncia que prevengan reabsorciones radiculares, lesiones de manchas blancas, caries, gingivitis y la pérdida de motivación por parte del paciente, las expectativas de los pacientes es que se complete en un corto período de tiempo y de manera eficiente, por lo que, la aceleración del movimiento dental ha ganado popularidad recientemente ${ }^{(5)}$.

Hasta la fecha, varios modelos de movimiento ortodóncico acelerado han sido reportados, entre ellos, farmacoterapia ${ }^{(6)}$, terapia de láser de baja intensidad ${ }^{(7)}$, campos de pulso electromagnéticos ${ }^{(8)}$, $\operatorname{corriente}$ eléctrica ${ }^{(9)}$, corticotomía $^{(10)}$, la vibración mecánica ${ }^{(11)}$, la piezoincisión ${ }^{(12)}$ y las microosteoperforaciones ${ }^{(13)}$. Estas últimas presentan un enfoque innovador, menos invasivo y más eficiente. Consiste en pequeñas y superficiales osteoperforaciones que pueden ser colocados de forma segura en la superficie vestibular o lingual de las tablas corticales por el ortodoncista tratante, con efectos secundarios leves y dolor o incomodidad limitados ${ }^{(14)}$. Tanto en estudios en animales como en humanos, la aplicación de osteoperforaciones poco profundas en la proximidad del diente en movimiento resulta en un incremento significativo de inflamación, activación osteoclástica, remodelación ósea y movimiento dentario(15).

La biomecánica es crucial en todos los aspectos de la odontología, especialmente en ortodoncia. Después de la aplicación de una fuerza externa inapropiada a la cavidad oral, el desarrollo de un campo de estrés en los tejidos de soporte generalmente conduce a un resultado fallido. El análisis de elementos finitos (AEF) es un instrumento matemático útil para la ortodoncia y puede determinar la cantidad de tensión, compresión y desplazamiento en el complejo dentoalveolar debido a las diferentes condiciones de carga de la fuerza. El AEF se ha convertido en una herramienta poderosa para la investigación biomecánica dental debido a su mayor disponibilidad, capacidad y facilidad de uso de software en modelos biológicos. Este análisis es considerado efectivo y no invasivo(16).

Así, el propósito del presente estudio fue investigar biomecánicamente si el tratamiento con micro-osteoperforaciones (Mops) genera diferencias de desplazamiento y de tensiones a nivel óseo cuando se aplica una carga ortodóncica, mediante el uso de análisis de elementos finitos.

\section{MATERIAL Y MÉTODOS}

Un modelo de mandíbula dentada obtenida de un banco de datos fue utilizado para el análisis. En este modelo se eliminó el segundo premolar mediante el software Catia (V5R16), para crear la región a estudiar. Este modelo consideró un grosor del hueso cortical de $2 \mathrm{~mm}$ que rodea el hueso trabecular. Se diseñó manualmente una banda a nivel molar con un tubo de 0,022 de altura $\times 0.028$ de ancho y $4 \mathrm{~mm}$ de largo, brackets con slot de dimensiones similares y un arco de acero de sección rectangular de $0.019 \times 0.025 \mathrm{~mm}$. Posteriormente, se dividieron en 3 modelos dependiendo de la posición de las Mops (Fig.1). Estas Mops fueron de $1.5 \mathrm{~mm}$ de diámetro $\times 5 \mathrm{~mm}$ de profundidad abarcando hueso cortical y trabecular, siendo tres a nivel vestibular, tres por lingual y una a nivel oclusal.

La simulación se realizó exportando los modelos al software de análisis de elementos finitos Ansys Workbench versión 19.0 (Ansys Inc., Canonsburg, PA, EE. UU.), y las estructuras para el análisis se consideraron isotrópicas, homogéneas y linealmente elásticas. El módulo de elasticidad y el coeficiente de Poisson se obtuvieron de la literatura (Tabla 1). Para simplificar el análisis del software, se utilizó una hemimandíbula, aplicando la función espejo con un plano de simetría sagital que atraviesa el área mesial de los incisivos centrales inferiores y a nivel de la sínfisis mandibular.

Para la simulación, se aplicó una carga estática horizontal de $150 \mathrm{gr}$ $(1,5 \mathrm{~N})$, simulando un resorte cerrado de Nitinol, tanto a nivel molar en dirección mesial como a nivel interproximal entre canino e incisivo lateral en dirección distal. Todos los contactos fueron considerados como 100\% unidos. El número de elementos fue de 423.900 y los nodos promedio fueron de 264.062.

Las tensiones analizadas correspondieron a tracción y compresión a nivel óseo, junto con el desplazamiento total de cada modelo.

Los modelos se estudiaron como un sistema completo con todas las escalas gráficas estandarizadas y se compararon entre ellos mediante la visualización de mapas de colores.
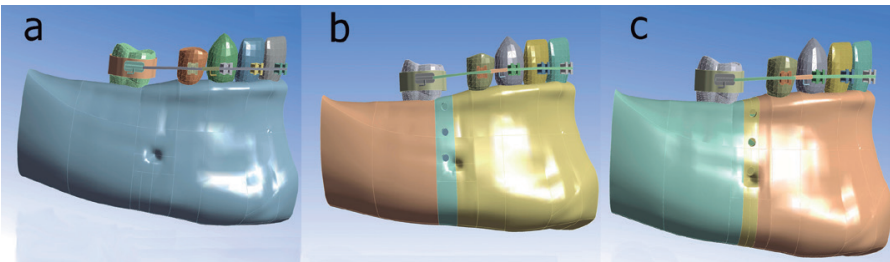

Figura 1. a) Sin Mops (control); b) Mops adyacentes a $1 \mathrm{~mm}$ del primer molar; c) Mops a $4 \mathrm{~mm}$ del molar (en el centro entre molar y premolar).

Tabla 1: Propiedades mecánicas de los materiales.

\begin{tabular}{|c|c|c|c|}
\hline Material & $\begin{array}{c}\text { Young } \\
\text { modulus } \\
(\mathrm{GPa})\end{array}$ & $\begin{array}{l}\text { Poisson } \\
\text { coefficient }\end{array}$ & References \\
\hline Hueso cortical & 13.4 & 0.3 & Feng y col. ${ }^{(17)}$ \\
\hline Hueso trabecular & 1.4 & 0.3 & Ozdemir Dogan ${ }^{(18)}$ \\
\hline $\begin{array}{l}\text { Hueso cortical } \\
\text { perforación }\end{array}$ & 11.1 & 0.3 & Zang y col.(19) \\
\hline $\begin{array}{l}\text { Hueso trabecular } \\
\text { perforación }\end{array}$ & 1.11 & 0.3 & Zang y col.(19) \\
\hline Diente & 20.3 & 0.3 & Feng y col.(17) \\
\hline Brackets y banda & 205 & 0.3 & Feng y col.(17) \\
\hline
\end{tabular}

\section{RESULTADOS}

La tabla 2 resume los resultados observados en este estudio.

Existe ligera tendencia a aumentar el desplazamiento del molar con la presencia de Mops, siendo levemente mayor en el molar que presenta las perforaciones más adyacentes; sin embargo, estos valores son muy próximos entre sí.

La zona de tracción en el hueso se aprecia a nivel cortical de la región cervical distal y tiende a aumentar sin la presencia de Mops. (Fig. 2 a, c, e)

La zona de compresión ósea se encuentra a nivel cortical de la región cervical mesial y muestra una tendencia a aumentar con la presencia de perforaciones adyacentes al molar. ( Fig. 2 b, d, f)

Tabla 2: Desplazamiento total y tensiones a nivel molar y óseo en los diferentes modelos analizados.

\begin{tabular}{l|c|c|c}
\hline & $\begin{array}{c}\text { Desplazamiento } \\
\text { (um) }\end{array}$ & $\begin{array}{c}\text { Tracción } \\
\text { hueso (MPa) }\end{array}$ & $\begin{array}{c}\text { Compresión } \\
\text { hueso (MPa) }\end{array}$ \\
\hline $\begin{array}{l}\text { Modelo 1 } \\
\text { (control) }\end{array}$ & 0,117 & $0,16^{*}$ & 0,3 \\
\hline $\begin{array}{l}\text { Modelo 2 } \\
\text { (adyacente) }\end{array}$ & 0,123 & $0,21^{*}$ & $0,40^{* *}$ \\
\hline $\begin{array}{l}\text { Modelo 3 } \\
\text { (centro) }\end{array}$ & 0,119 & $0,21^{*}$ & $0,28^{* *}$ \\
\hline $\begin{array}{l}\text { * dirección distal } \\
\text { ** dirección mesial }\end{array}$
\end{tabular}

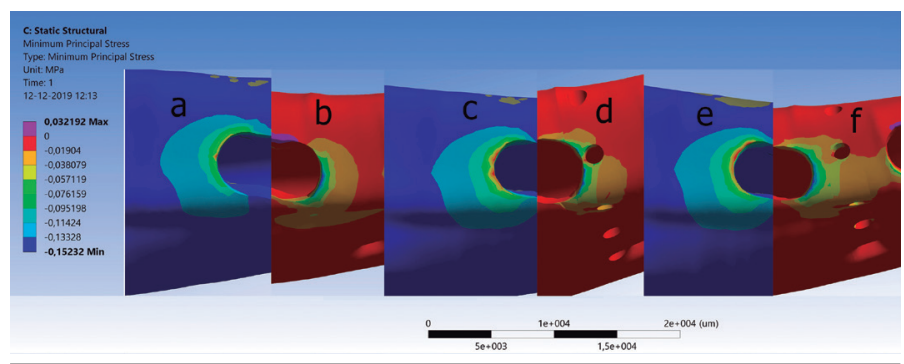

Figura 2. a) tracción ósea modelo 1, b) compresión ósea modelo 1, c) tracción ósea modelos 2 , d) compresión ósea modelo 2, e) tracción ósea modelo 3 , f) compresión ósea modelo 3. 


\section{DISCUSIÓN}

El objetivo de este estudio fue evaluar biomecánicamente si el tratamiento con Mops genera diferencias de desplazamiento y de tensiones a nivel óseo cuando se aplica una carga ortodóncica. Una de las teorías, es que las Mops pueden variar el módulo elástico del hueso adyacente y permitir mayor libertad de movimientos de tracción y compresión ${ }^{(2)}$. Esto se justifica ya que las propiedades del material, en nuestro modelo el tejido óseo cortical, pueden influir considerablemente en la distribución de tensiones y deformaciones de una estructura ${ }^{(24)}$.

Dentro de los resultados obtenidos, a pesar que se observó una ligera tendencia a aumentar el desplazamiento del molar con la presencia de Mops, no existieron mayores variaciones en relación a las magnitudes de desplazamiento ni tensiones entre los diferentes modelos. Al parecer, el uso de Mops, que podría influir en disminuir la resistencia o módulo elástico del hueso adyacente, éste parece no ser relevante, ya que no se observan mayores variaciones desde el punto de vista biomecánico. Es importante resaltar que cuando las Mops se localizan a $>3 \mathrm{~mm}$ de diente a mover, parece carecer de influencia en la densidad ósea, ni en la velocidad de movimientos dentarios ${ }^{(21)}$, Lo anterior, podría explicar los modelos donde las Mops se realizaron a $4 \mathrm{~mm}$ en el presente estudio, pero no justificaría aquellos donde se realizaron las perforaciones a $1 \mathrm{~mm}$ de distancia del molar.

Existen estudios de AEF en maxila con resultados favorables al uso de estimulación ósea ${ }^{(22,23)}$; sin embargo, no encontramos estudios en mandíbula, por lo que los resultados discretos de este estudio podrían explicarse por la diferencia en la densidad ósea de ambos maxilares.

El posible efecto de las Mops puede ser explicado desde el punto de vista biológico, ya que se ha relatado que al aplicar microosteoperforaciones, es posible que se incremente el número de osteoclastos significativamente a lo largo de la ruta de movimiento, lo que aumentaría la tasa de reabsorción ósea y la velocidad de movimientos dentarios ${ }^{(13)}$. Los movimientos dentarios implican señales específicas que van desde el ligamento periodontal hasta el hueso alveolar. Estas señales, que son citosinas inflamatorias y quimiocinas que se generan desde el hueso adyacente al diente en movimiento junto con el sitio donde se realizan las Mops, podrían influir en el proceso de remodelación ósea alveolar y aumentar la velocidad de movimiento dentario(20,14), lo cual no puede ser evaluado mediante la metodología utilizada en este estudio.

Todas las magnitudes de tensiones de compresión y tracción a nivel óseo identificadas en este estudio se encontraron dentro del rango fisiológico tolerable por el hueso circundante ${ }^{(24)}$.

Finalmente, a pesar de que existen contradicciones en relación a la efectividad de las Mops en los movimientos dentarios ${ }^{(14,25)}$, este estudio demostró que desde el punto de vista biomecánico no existen diferencias evidentes en los valores de tensiones ni de desplazamiento entre los modelos analizados. Así, es necesario estudiar la influencia de las Mops del punto de vista biológico para finalmente definir su verdadero efecto en los movimientos dentales.

\section{CONFLICTO DE INTERÉS}

Los autores declaran no tener conflicto de interés comercial con las empresas de software u otras que comercializan productos que realizan Mops.

\section{Bibliografía}

1. O'Connor JA, Lanyon LE, MacFie $H$. The influence of strain rate on adaptative bone remodeling. J Biomech. 1982; 15(10):767-81.

2. Alikhani M. Clinical Guide to Accelerated Orthodontics: Whit a focus of microosteoperforations. Cham: Springer International Publishing AG; 2017.

3. Domínguez $\mathrm{C}$, Velásquez $\mathrm{C}$. Reevaluation of the Strain Pressure Theory Toward a better understanding the biology of tooth movement. Int J Odontostomat. 2017; $11(2): 133-40$

4. Taddei SR, Andrade I Jr, Queiroz-Junior CM, Garlet TP, Garlet GP, Cunha Fde Q, et al. Role of CCR2 in orthodontic tooth movement. Am J Orthod Dentofacial Orthop. 2012:141:153-60.

5. Pasaoglu A. Effects of mechanical vibration on miniscrew implants and bone: Fem analysis. Int Orthod. 2019;17:38-44.

6. Hashimoto F, Kobayashi Y, Mataki S, Kobayashi K, Kato Y, Sakai H. Administration of osteocalcin accelerates orthodontic tooth movement induced by a closed coil spring in rats. Eur J Orthod. 2001;23(5):535-45.

7. Doshi-Mehta G, Bhad-Patil WA. Effficacy of low-intensity laser therapy in reducing treatment time and orthodontic pain: a clinical investigation. Am J Orthod Dentofacia Orthop. 2012;141(3):289-97.

8. Stark TM, Sinclair PM. Effect of pulsed electromagnetic fields on orthodontic tooth movement. Am J Orthod Dentofacial Orthop. 1987:91(2):91-104.

9. Davidovitch Z, Finkelson MD, Steigman S, Shanfeld JL, Montgomery PC Korostoff E. Electric currents, bone remodeling, and orthodontic tooth movement II. Increase in rate of tooth movement and periodontal cyclic nucleotide levels by combined force and electric current. Am J Orthod. 1980;77(1):33-47.

10. Peron AP, Johann AC, Papalexiou V, Tanaka OM, Guariza-Filho O, Ignácio $\mathrm{SA}$, et al. Tissue responses resulting from tooth movement surgically assisted by corticotomy and corticision in rats. Angle Orthod. 2017;87(1):118-24.

11. Alikhani M, Lopez JA, Alabdullah H, Vongthongleur T, Sangsuwon C, Alansar $S$, et al. High-Frequency acceleration: therapeutic toll to preserve bone tooth extractions. J Dent Res. 2016;95(3):311-18.

12. Aksakalli S, Calik B, Kara B, Ezirganli S. Accelerated tooth movement with piezocision and its periodontal-transversal effects in patients with Class II malocclusion. Angle Orthod. 2016;86(1):59-65.

13. Alikhani M, Alansari S, Sangsuwon C, Chou MY, Alyami B, et al. Microosteoperforations: Minimally invasive accelerated tooth movement. Semin Orthod 2015;21:162-9

14. Alikhani M, Raptis M, Zoldan B, Sangsuwon C, Lee Y, Alyami B, et al. Effect of micro-osteoperforations on the rate of tooth movement. Am J Orthod Dentofac Orthop. 2013; 144(5):639-48.

15. Teixeira CC, Khoo E, Tran J, Chartres I, Liu Y, Thant LM, et al. Cytokine expression and accelerated tooth movement. J Dent Res. 2010;89:1135-41.

16. Xue J, Ye N, Yang X, Wang J, Wang Y, Li J, et al. Finite element analysis of rapid canine retraction through reducing resistance and distraction. J Appl Oral Sci. 2014;22(1):52-60

17. Feng Y, Kong WD, Cen WJ, Zhou XZ, Zhang W, Li QT, et al. Finite element analysis of the effect of power arm locations on tooth movement in extraction space closure with miniscrew anchorage in customized lingual orthodontic treatment. Am J Orthod Dentofacial Orthop. 2019;156(2):210-19.

18. Özdemir Doğan D, Polat NT, Polat S, Şeker E, Gül EB. Evaluation of "all-on-four" concept and alternative designs with 3D finite element analysis method. Clin Implant Dent Relat Res. 2014;16(4):501-10.

19. Zhang X, Li XW, Li JG, Sun XD. Preparation and mechanical property of a novel 3D porous magnesium scaffold for bone tissue engineering. Mater Sci Eng $C$ Mater Biol Appl. 2014 Sep;42:362-7.

20. Aboalnaga A, Salah M, El-Ashmavi N, Soliman S. Effect of micro-osteoperforation on the rate of canine retraction: a splint-mouth randomized controlled trial. Prog Orthod. 2019;20(1):21.

21. Cramer C, Campbell P, Opperman L, Tadlock L, Buschang P. Effects of microosteoperforations on tooth movement and bone in the beagle maxilla. Am J Orthod Dentofacial Orthop. 2019;155(5):681-92.

22. Yang C, Wang C, Deng F, Fan Y. Biomechanical effects of corticotomy approaches on dentoalveolar structures during canine retraction: a 3-dimensional finite element analysis. Am J Orthod Dentofacial Orthop. 2015 148(3):457-65

23. Gupta S, Ahuja S, Bhambri E, Jaura BS., Ahuja V. Three-dimensional finite element analysis to evaluate biomechanical effects of different alveolar decortication approaches on rate of canine retraction. Int Orthod. 2019;17(2):216-26.

24. Bozcaya D, Muftu S, Muftu A. Evaluation of load transfer characteristics of five different implants in compact bone at different load levels by finite elements analysis. J Phrosthet Dent. 2004;92(6):523-30.

25. Alkebsi A, Al-Maaitah E, Al-Shorman H, Abu-Alhaija E. Three- dimensional assessment of the effect of micro-osteoperforations on the rate of tooth movement during canine retraction in adults with Clasa II malocclusion: A randomized controlled clinical trial. Am J Orthod Detofacial Orthop. 2018;153(6):771-85. 